\title{
A Modified FFT Algorithm for OFDM Based Wireless System
}

\author{
G. Harish Kumar, S. Srikanth
}

\begin{abstract}
The constrained open range and the wastefulness in the range use in a fixed range task strategy, requests another correspondence model to abuse the present remote range unquestionably. This new structures connection point of view is accumulated as bleeding edge sifts through moreover as Dynamic Spectrum Access (DSA) and shrewd radio systems. The FFT and its turn IFFT are fundamental estimations in signal overseeing, programming portrayed radio, and the most encouraging change framework for example Symmetrical OFDM. From the normal organization of OFDM we can locate to FFT/ IFFT modules expect the essential work for any OFDM based handset. So when zero respected wellsprings of data/yields overpower nonzero inputs/yields, by then wide IFFT/FFT estimation for OFDM is never again supportive in term of implementation moment It is conceivable to decrease the implementation moment with "pruning" the FFT. In this we have finished a novel and significant data zero searched for after FFT pruning (IZTFFTP) estimation subject to DIF radix-2 framework. Separate from different estimations, the yielded outcome of IZFFTP show that it is free of condition of the zero respected data what's everything the all the more keeping up a reasonable exchange off among reality bizarreness of any structure by not just diminishing the extent of complex extension correspondingly a comparative number of sided advancements what's more. The proposed join is executed in essential level PC program for example in $C++$ and this takes after the Cooley-Tukey radix-2 FFT estimation, holding all the key highlights, for example, straightforwardness and consistency, by making some assortment and programming adjustment
\end{abstract}

Overview Terms-Cognitive radio, OFDM, FFT, Pruning Techniques, Execution time.

\section{INTRODUCTION}

$\mathrm{F}_{\text {FT }}$ be a focal contraption in field of sign controlling \& direct structure assessment. DFT isn't spread out \& utilized all around until FFT was planned. In any case, trademark peculiarity among FFTs range targets and computational moment use obliges its request. To plan with the referencing or need of a structure, the standard system is to build up the data improvement $\mathrm{x}(\mathrm{n})$ by padding number of zeros toward its peak and which is in danger for a general clearing estimation of computational time. Regardless, figuring on undesired repeat is vain. As the OFDM based informational radio [1] can pound unequivocal sub transporters to keep up a basic less dreadful ways from mess up for the empowered customer. Fittingly, that there could be a titanic number of zero regarded information sources/yields stand pulled once again from non-zero terms. This is the most

Revised Manuscript Received on December 30, 2019.

G. Harish Kumar, Assistant Professor, MRECW

,harish.gopadi@gmail.com

S. Srikanth,Assistant Professor, MRECW

srimrecw@gmail.com fundamental thing in the reasonable surprising OFDM, which is use since baseband transmission in run pooling structure. Regardless of the course by which that goliath exchange speed reinforces high data rates yet in each obliging sense it is unnoticeable neighboring void information move limit. So much persuading data rates are made by using non-arriving at void subcarriers of a concentrated on run pool. This kind of OFDM is known as non-coming to NC-OFDM [2-3] or OFDM, which keeps up a key OK ways from the hazardous changing activity by deactivating those subcarriers, which are gotten by different saw customers. That gathers the data estimations of the IFFT"es of those particular subcarriers is zero. As NC-OFDM wires titanic number of de-affected or invalid subcarrier for instance degrees of zero regarded wellsprings of data/yields win non-zero data sources/yields. So the standard FFT is never again pleasing to the degree multifaceted nature, execution time and apparatus filtering through. Several specialists have proposed disengaging way to deal with oversee manage control regulate direct make FFT snappier by "pruning" the standard one [4].

In this we contain planned an information 0 sifted for after IZTFFTP figuring, sensible for NC-OFDM based handset. It unending store of the creation line Cooley-Tukey radix-2 DIF estimation with structure factorizing procedure outcome show IZTFFTP is extra fit than standard FFT. This is made in going with sub sections: area II looks gets a few information about in this subject, zone III contain organization of NC OFDM, part IV examines for after regarding general FFT figuring (using structure factorisation framework), separate $\mathrm{V}$ joins the planned system for pruning (checking flowchart), results and wrapping up comments are showed up in pack VI\&VII wholeheartedly.

\section{RELATED RESEARCH}

As FFT contain a few fundamental applications in field of sign controlling, different reviews are so far proceeding to make it reliably fit and adaptable to the degree framework key. So as near reduce check occasion of common FFT estimation, a few pruning figuring contain be planned. In the 1971 J.D Markel propose initial FFT pruning check .FFT depended upon FFT DIF model. Following close to method Skinner [5] have planned an estimation subject to FFT DIT model utilizing the information sources focuses as it's been said. By at that point, checking both the data and yield Srinivas and pruning Rao contain planned an extra algorithm[6].In 2000 R.G.Alves gave a thought for general FFT pruning algorithm[7].A clear perspective on FFT estimation, convert ruin system include be planned with Sorensen and Burrus [8].

TD strategy everything considered a changed capability in CTFFT figuring, there DFT is restricted keen on 2 persistently little DFTs.

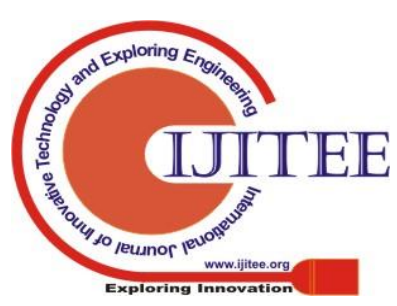


Notwithstanding how it is dumbfounding in any case to the degree gear use TD is constantly stunning and versatile quite than prune.

From these we find various thought concerning necessities of an fitting pruning framework \& their way to deal with oversee control direct prune a FFT. A colossal piece of them depends upon the diagram of a structure (neighboring TD), contains $\mathrm{N}$ lines $\& \log 2 \mathrm{~N}$ isolates. In any case, this age of a right hand pull it together is a tangled and dull system and difficult to see as well. To find free sour as of these Inefficiencies we contain planned IZTFFTP check, which is the mind boggling separation when all is said in done Coole-Tukey estimation (utilizing cross zone factorization) [9], by basic varying in the programming move toward.

\section{OFDM STRUCTURE}

OFDM be an advanced several transporter rule system. OFDM utilizes interminable genuinely pulls back even subcarrier.This subsection we motivation talk regarding OFDM structure \& its age method utilizing common diagram in figure 1-(A, B).mostly in DSA sort out, it isn't all around that truly matters conceivable to discover a wrapping square of range, these are used completely. thus with utilizing active range seeing \& canal evaluation framework, the sub carriers to are gotten to with understood client butchered. The OFDM based handsets folks set up to disable pre-owned sub carriers be identified because NC-OFDM based handsets [3].by Fig.1 we resolve take a gander at inside masterminding of this unimaginable structure a piece immediately.

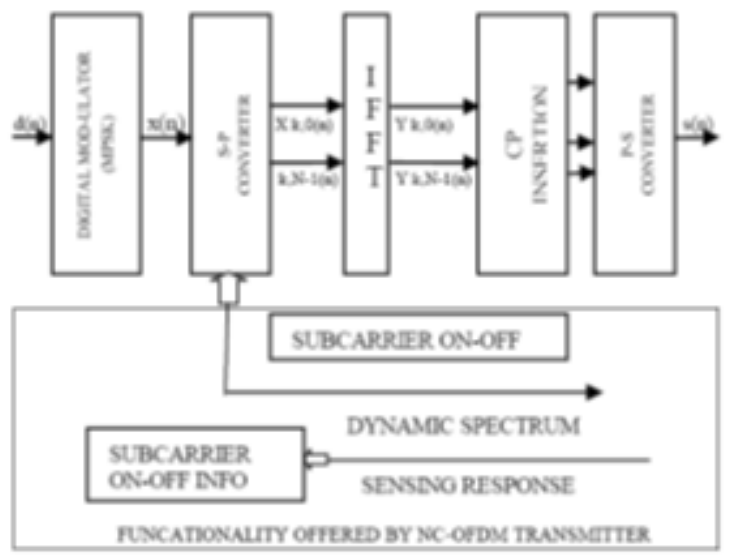

Fig.1-A: General NC-OFDM transmitter

Let $\mathrm{x}(\mathrm{n})$ is changed sort of information flow $\mathrm{d}(\mathrm{n})$. accent have be complete by several robotized change plot. By after that reasonable information flow is allocated addicted to $\mathrm{N}$ divide information stream with uses S-P converter. The aggregate of streams is transmit during folks better than commonplace sub carriers $\&$ in the wake of including them we resolve find compound OFDM sign. As we broke down before to NC-OFDM handset contain lots of deactivate sub carriers to used with got customers. individuals sign be in like way transmit to beneficiary yet contain rejection goliath commitment in FFT/ IFFT figuring. To diminish spread picture the starting point cyclic prefix (CP) square is added to the picture \& this CP fills in as gatekeeper break among 2 unmistakable picture. By next it is experienced P-S converter. To conclude the sign is up sample plus experienced a D/A converter for changing over it keen on clear sign $\mathrm{s}(\mathrm{n})$ plus transmit through RF disturb.

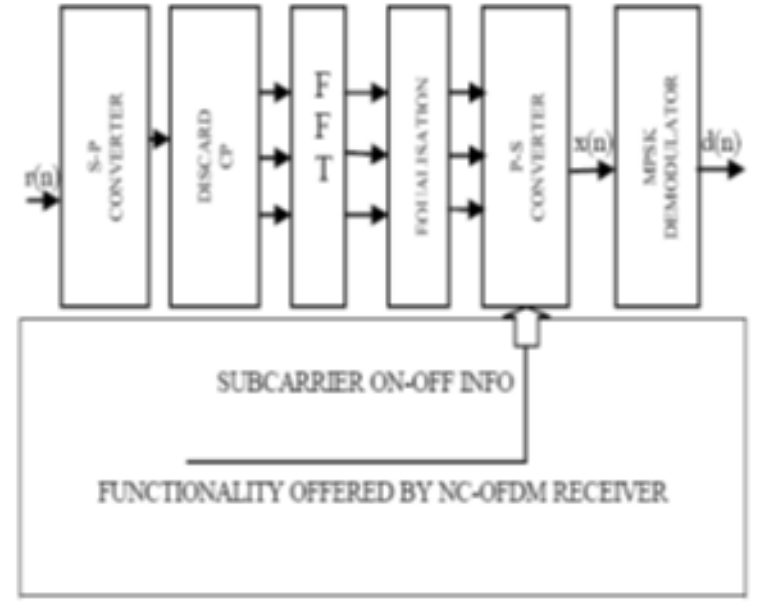

Fig.1-B: General NC-OFDM receiver

At the gatherer $s(n)$ is changed over to mechanized sign $r(n)$ by experiencing a $\mathrm{A} / \mathrm{D}$ converter. By then the sign is experienced a S-P converter to make parallel data stream .After this CP is discarded and urged that sign to the FFT square to change the time zone data into accentuate space. Finally to get the critical sign, multiplexing using P-S converter and demodulation has been done.
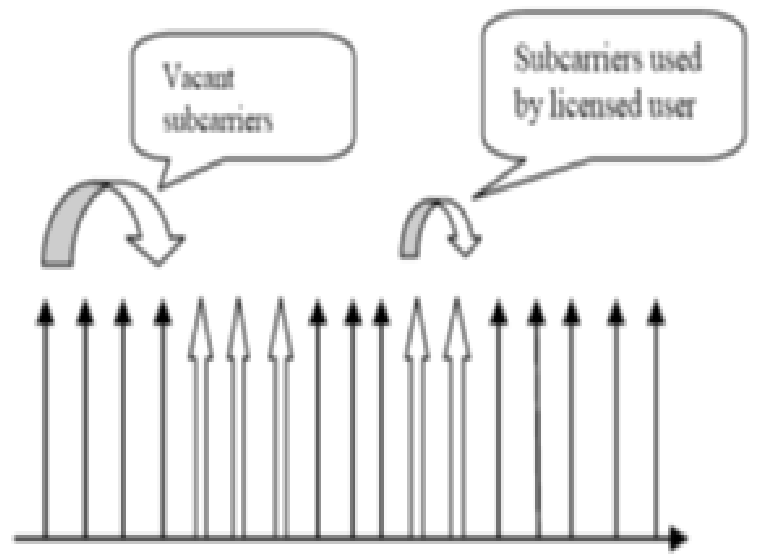

Fig.2: subcarrier distribution in NC-OFDM system

\section{GENERAL FFT ALGORITHM}

The FFT/IFFT is the most basic bit of any sign getting ready structure. now too in OFDM base handset individuals are mainly computational raised squares of unmitigated structure. So a incompetent IFFT/FFT can diminish general structure retort. This relies upon radix-2 DIF FFT figuring, which contain a gathering \& whipping way of thinking, where N-centers DFT is crushed into reliably little DFTs(with odd and even part overwhelmingly).

The essential condition for DFT is-

$$
\mathrm{X}(\mathrm{n})=\sum_{m=0}^{N=1} X_{0}(K) e^{-\frac{3 j i m k}{N}} \quad \mathrm{n}=0,1,2 \ldots \mathrm{N}-1 \ldots \ldots \text { (1) }
$$

$\mathrm{N}$ is the order,for model if $\mathrm{N}=4$, by then we can create the eqn-1 out of a system structure 


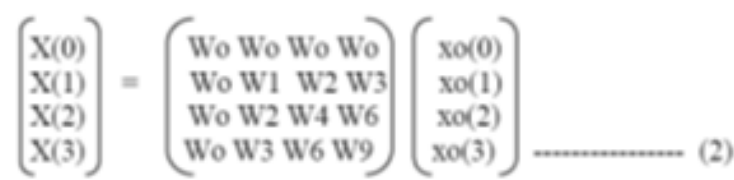

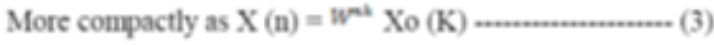

as-

As W (address squirm factor) \& $\mathrm{Xo}(\mathrm{K})$ are extraordinary, thus to unravel eqn (3), complex expansion \& $\mathrm{N}(\mathrm{N}-1)$ complex progress is necessary. Regardless, FFT estimation diminishes computational occasion by lessening all out no.of complex growthes \& augmentations.

To structure FFT estimation, genially I take $\mathrm{N}=$, where $\square$ is a whole number and upgrading the eqn (2) as
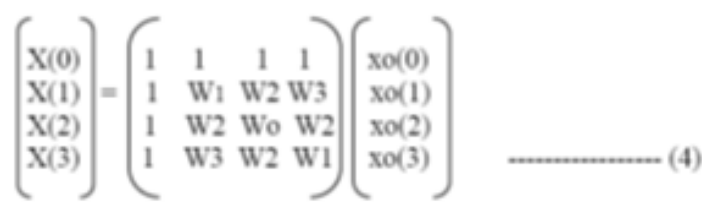

So currently with calculating system (4) \& changing solicitation for rows:

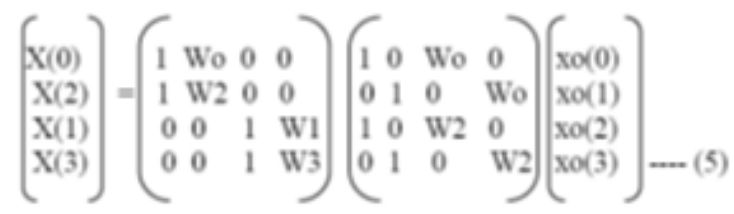

In spite of how it is a multifaceted point of view to find regardless it is in no way, shape or form hard to complete in a PC program. Thusly we kept up this methodology for FFT computation. So, FFT estimation is an noteworthy considering course of action of $\mathrm{N} \times \mathrm{N}$ structure addicted to $\mathrm{N}$ matrices (each $\mathrm{N} \times \mathrm{N}$ ). Fat continuously noticeable estimation of $\mathrm{N}$,we need to unwind this structure factorization procedure addicted to a graphical way which resolve put up a flow chart pro a skilled PC agenda. We contain indicated a graphical outlook in fig-(3) someplace $\mathrm{N}=8$.

Unfaltering stream diagram relies upon two parent conditions, that are-

$$
\begin{aligned}
& x_{l}=x_{l-1}(K)+W^{p} x_{l-1}\left(K+\frac{*^{i}}{2^{l}}\right) \\
& x_{l}\left(K+\frac{N}{2^{l}}\right)=x_{l-1}(K)-W^{p} x_{l-1}\left(K+\frac{N}{2^{l}}\right)
\end{aligned}
$$

Where $1=$ no. Of array in the flow graph, and $\mathrm{W}^{\mathrm{P}}$ is the twiddle factor.

$$
\text { Those two points } \mathrm{x}_{1} \&^{x_{l}\left(K+\frac{N}{2}\right)} \text {, secluded by }
$$
N/2,are known as twofold obsession to each other. Each twofold center point needs single consider both the conditions have same information stream and the estimation of squirm factor is other than same. The standard separate is the purpose of repression $\mathrm{WP}$ of the as $\mathrm{WP}=-\mathrm{WP}+\mathrm{N} / 2$.with this approach condition we register FFT for to exacting model $(\mathrm{N}=4)$,

Then

$$
\begin{aligned}
& \odot=, 0^{e e} \text { operation [full pruning] } \\
& \mathbf{0}=\text { single operation [partial pruning] } \\
& \bigcirc=\text { full butterfly operation [no pruning] }
\end{aligned}
$$

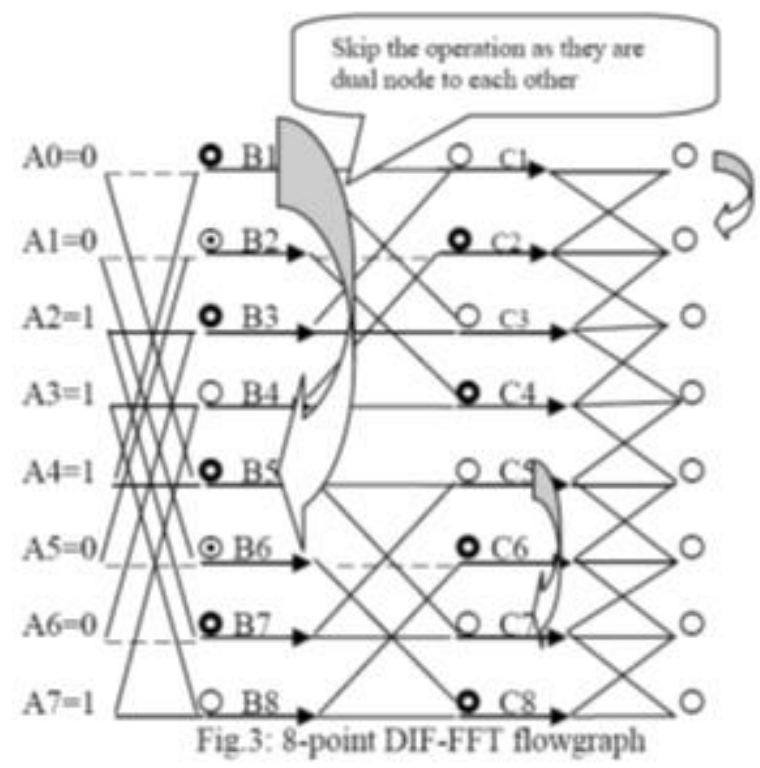

Just $\mathrm{NP} / 2=4$ compound increment \& $\mathrm{NP}=8$ compound improvement is required, which is strikingly not as much of help DFT framework. Regardless at the same time standard FFT will work wastefully when perpetual zero respected information sources are available show up contrastingly in relationship with non-zero data sources. To reduce this wastefulness in this I include planned a few methodologies \&change the ordinary flow chart [9]. In like way I contain planned another stream plot \& complete to a PC agenda

\section{PROPOSED PRUNING TECHNIQUES}

To build up point confinement of the FFT framework two or three pruning and clear various systems include planned by various specialists. In this, we contain understood another pruning structure for instance IZTFFTP by direct adjustment $\&$ a few change in standard flowchart of FFT [9] what's more joins some flawed numerical techniques to lessen the full scale execution time.

Zero after as in wide band correspondence structure an animal zone of repeat channel may be empty by the certified customer, so no. of zero regarded data sources are generously more undeniable than the non-zero regarded duties to a FFT/IFFT progress at the handset. By then this check will give best response to the degree reduced execution time by

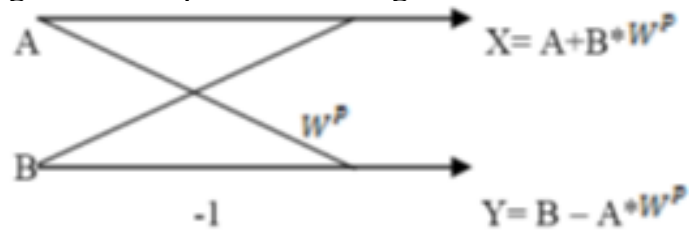

Fig .4: small butterfly unit of a dual node pair decreasing the no. of complex estimation required for squirm factor count. IZTFFTP have a solid looking through condition, which have a 2-D show up for dealing with the information and yield respects after each complement of butterfly count. In an information looking through outcome at whatever point it discovered ,zero ${ }^{\text {ee }}$ at any data, essentially square that check with allowing for after 2 fulfilling state:

Half Butterfly estimation or fragmentary pruning-The essential 


\section{A Modified FFT Algorithm for OFDM Based Wireless System}

check some bit of FFT is butterfly count. fig. 3 we choose a particular piece of a normal butterfly unit. An and B 2 compound information sources be twofold fixation just before one another .A filled butterfly check require 4 compound increases \& 6 composite structures/.

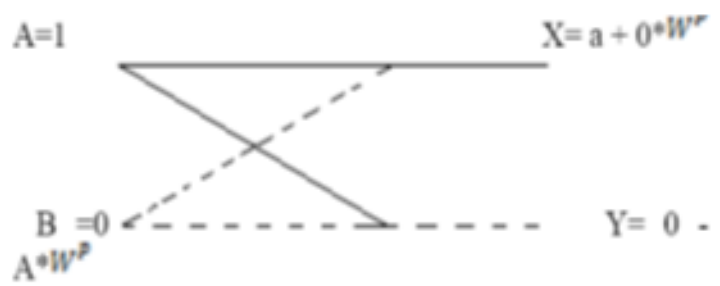

Fig 5: partial pruning structure

"0" development or complete the route toward pruning shows piece someplace both the commitment of twofold focus point pair is 0 . by yields got since the sensible check condition six-seven is in like way 0 .

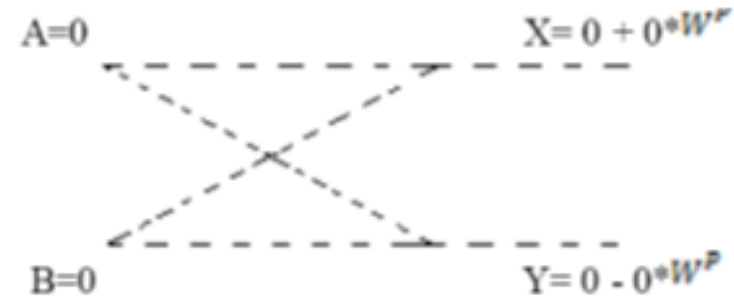

Fig.6: complete pruning structure

In this conditions where no. of 0 is strikingly goliath IZTFFTP mechanism mainly viably \& agenda resolve routinely go to going with focus point for required check discarding these superfluous complex estimations.

Along these lines the full scale number of compound extensions \& headways are compact strikingly proportionally as completing occasion what's more. In going with effect zone we resolve discover how fresh approach give improved reaction concerning general FFT check.

\section{RESULTS}

Accurately when all is said in done by a wide margin most by a wide edge of FFT or prune estimations be truly planned. several of them be done also in FORTRAN or in MATLAB. standard goals of individuals structure are to, they be not sensibly gainful sufficient data dataset. It is especially key to discover a figuring finished in raised level PC program, which can show the central guaranteed execution time for a FFT improvement. Here we have executed a FFT pruning structure in goliath level programming language for example in $\mathrm{C}++$ and execute it in Linux compose .To check the FFT results, before pruning development we have reproduced the center FFT code subject to framework factorization process, in a DSP territory for example VDSP++, which is the programming appraisal window of ADSP BF533 EZ-KITLITE DSP starter unit. The flowchart of the program has been appeared in figure-8.

Table-1 contains the ordinary information enlightening diagram, set away in a 2-D pack, mulling over generally veritable attributes $\&$ as this is just pro a NC - OFDM structure so level of conceivable unlicensed client isn't normally the staggering no of subcarriers.

\begin{tabular}{|c|c|c|}
\hline Input serial & Real part & Imaginary part \\
\hline$a[0]$ & 1 & 0 \\
\hline$a[1]$ & 1 & 0 \\
\hline$a[2]$ & 0 & 0 \\
\hline$a[3]$ & 0 & 0 \\
\hline$a[4]$ & 0 & 0 \\
\hline$a[5]$ & 0 & 0 \\
\hline$a[6]$ & 0 & 0 \\
\hline$a[7]$ & 1 & 0 \\
\hline
\end{tabular}

Table-1: 2-D array's input in tabular form

Expecting a[0], a[1] and a[7], these three wellsprings of data ought to be figured in a specific FFT improvement of mentioning 8 .

In table-2, the evaluation of the yields have been indicated expecting 1024 no of subcarriers are there in that OFDM structure.

\begin{tabular}{|c|c|c|c|c|c|c|}
\hline \multirow[t]{2}{*}{$\begin{array}{l}\text { Onder } \\
\text { of } \mathrm{FTT}\end{array}$} & \multicolumn{2}{|c|}{$\mathrm{Na} \mathrm{OfCM}^{*}$} & \multicolumn{2}{|c|}{$\mathrm{Na}$. of $\mathrm{CA}^{*}$} & \multicolumn{2}{|c|}{$\begin{array}{l}\text { execution time(mili } \\
\text { sesonds) }\end{array}$} \\
\hline & $\begin{array}{l}\text { Ondinary } \\
\text { FT }\end{array}$ & $\begin{array}{l}\text { Proued } \\
\text { FIT }\end{array}$ & $\begin{array}{l}\text { Ondinary } \\
\text { FFT }\end{array}$ & pried & centinary & mined \\
\hline 8 & 12 & 11 & 24 & 7 & 0.55 & 0.47 \\
\hline 16 & 32 & 25 & 88 & 23 & 0.76 & 0.68 \\
\hline 32 & 80 & 62 & 248 & 83 & 251 & 0.80 \\
\hline 64 & 192 & 132 & 632 & 207 & 9.41 & 1.80 \\
\hline 128 & 448 & 284 & 1528 & 511 & 21.02 & 2.65 \\
\hline 286 & 1024 & 584 & 3576 & 1127 & 77.02 & 3.91 \\
\hline 512 & 2304 & 1208 & 8185 & 2471 & 306.88 & 6.44 \\
\hline 1024 & 5120 & 2448 & 18424 & 5175 & 1204.32 & 13.97 \\
\hline
\end{tabular}

Yield shows the enormous decreasing of computational rash contemplations by reducing the firm no. of complex activity for example both the duplications and causes show to up contrastingly in relationship with the standard FFT development. Subsequently we have shown the thought concerning estimation of the decreased execution time for two evident checks (with pruning and without pruning) utilizing a relating planned $(2.10 \mathrm{GHz}$, Intel core 2 pair CPU) PC structure. 


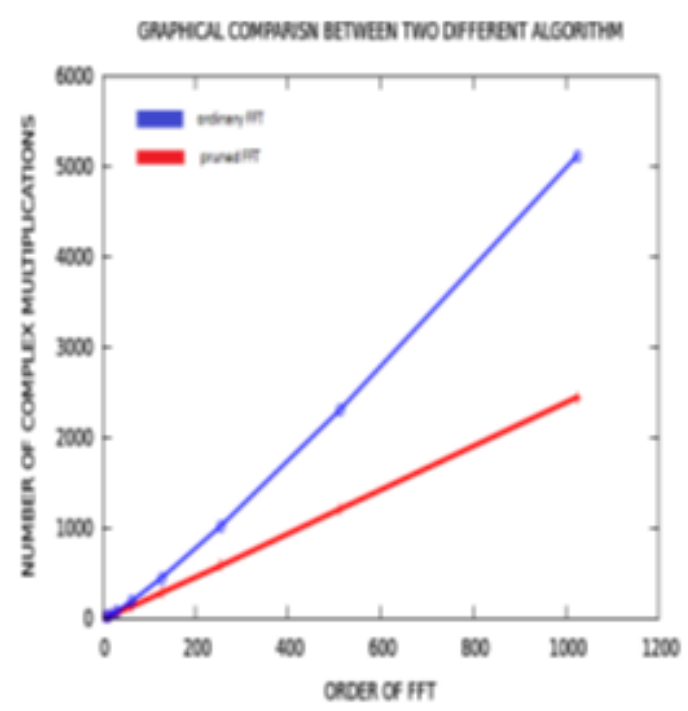

Fig.-7: graphical comparison between two different

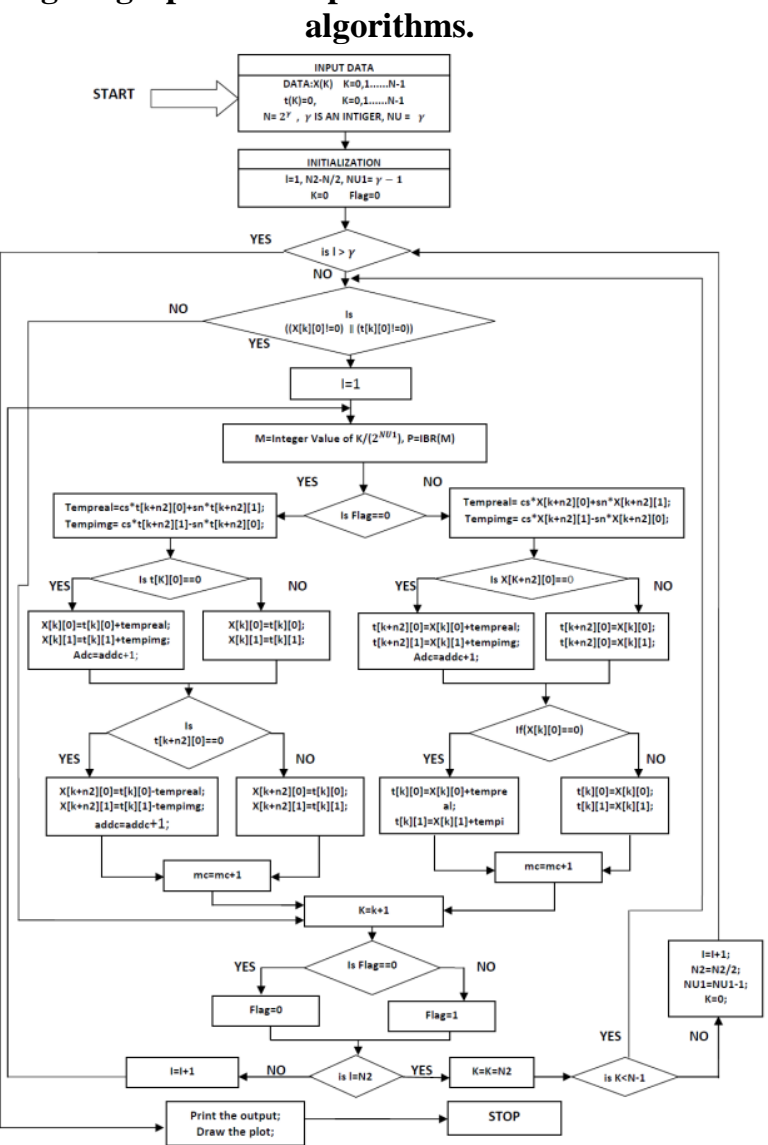

Fig.8- Programming Flowchart of the IZTFFTP algorithm

Utilizing Linux GNU plot we have shown the graphical appraisal of the yield reactions in Fig.- 7, where X-turn address the referencing for FFT and Y-focus point for the level of complex duplications.

\section{CONCLUSION}

In diagram through this paper we have proposed and finished a novel structure for FFT pruning estimation in raised level PC program by trading the set up join framework the major FFT figuring [9].Though by a wide edge a huge piece of the Researchers didn't give such an enormous measure of giganticness in the level of expansions yet it in like manner have a fundamental impact if there should be an occasion of equipment use (FPGA). Results shows IZTFFTP is a huge amount of incredible than traditional FFT consider it requires some hypothesis to pick where number of Zero respected wellsprings of information/yields are a more real need than the full scale number of non Zero terms, with keeping up a not very undesirable exchange off among reality multifaceted nature, and it is in like way self-coordinating to any information edifying blends.

\section{REFERENCES}

1. J. Mitola, III, "Cognitive Radio: An Integrated Agent Architecture for Software Defined Radio," Thesis (PhD), Dept. of Teleinformatics, Royal Institute of Technology (KTH), Stockholm Sweden, May 2000.

2. J. D. Poston and W. D. Horne, "Discontinuous OFDM considerations for dynamic spectrum access in idle TV channels," inProc. IEEE Int Symp. New Frontiers Dynamic Spectra. Access Networks, vol. 1, (Baltimore, MD, USA), pp. 607-610, Nov.

3. R. Rajbanshi, A. M. Wyglinski, and G. J. Minden, Cognitive Radio Communication Networks, ch. 5. Springer-Verlag, 2007.

4. J. D. Markel, "FFT Pruning," IEEE Trans. Audio Electroacoust., vol. 19, pp. 305 - 311, Dec. 1971.

5. D. P. Skinner, "Pruning the Decimation in time FFT algorithm," in Proc. IEEE Int. Conf.Acoust., Speech, Signal Process., vol. 24, Apr. 1976, pp. 193 - 194

6. T. V. Sreenivas and P. Rao, "FFT algorithm for both input and output Pruning," in Proc.IEEE Int. Conf. Acoust., Speech, Signal Process, vol. 27, June 1979 , pp. $291-292$

7. R. G. Alves, P. L. Osorio, and M. N. S. Swamy, "General FFT Pruning Algorithm," in Proc.43rd IEEE Midwest Symp. Circuits and Systems, vol. 3, Aug. 2000, pp. $1192-1195$.

8. H. V. Sorensen and C. S. Burrus, "Efficient computation of the DFT with only a subset of input or output points," IEEE Trans. Signal Processing, vol. 41, pp. 1184 - 1200, Mar. 1993.

9. E. Oran. Brigham, "The Fast Fourier Transform and Its Applications" Prentice Hall Publication, 1988, ISBN: 0133075052 